\title{
Traductions et adaptations françaises de l'Elegia di madonna Fiammetta
}

Serge Stolf

\section{OpenEdition}

\section{Journals}

Édition électronique

URL : http://journals.openedition.org/cei/898

DOI : $10.4000 /$ cei.898

ISSN : 2260-779X

\section{Éditeur}

UGA Éditions/Université Grenoble Alpes

\section{Édition imprimée}

Date de publication : 15 juillet 2008

Pagination : 177-194

ISBN : 978-2-84310-122-9

ISSN : 1770-9571

\section{Référence électronique}

Serge Stolf, "Traductions et adaptations françaises de l'Elegia di madonna Fiammetta », Cahiers d'études italiennes [En ligne], 8 | 2008, mis en ligne le 15 janvier 2010, consulté le 01 mai 2019. URL: http://journals.openedition.org/cei/898; DOI : 10.4000/cei.898 


\title{
TRADUCTIONS ET ADAPTATIONS FRANÇAISES \\ DE L'ELEGIA DI MADONNA FIAMMETTA
}

\author{
Serge Stolf \\ Université Stendhal-Grenoble 3
}

L'ouvrage de Boccace qui a connu et connaît le plus grand nombre de traductions françaises est sans conteste le Décaméron. Est-ce à dire que cette curiosité presque exclusive n'a laissé, en France, aucune place pour d'autres œuvres du conteur florentin? Il suffit de parcourir les éditions lues au XV siècle et au siècle suivant pour apporter une réponse plus nuancée. C'est aux traductions et adaptations françaises de l'Elegia di madonna Fiammetta au XVI siècle que nous consacrerons ici la première partie de ces quelques réflexions et mises au point.

$S^{\prime}$ il faut bien admettre que la notion de traduction ne reposait pas alors sur les mêmes exigences de fidélité que de nos jours, il n'en reste pas moins légitime de faire la part entre ce qui relève de la traduction, malgré toute sa relative désinvolture envers le texte original, et l'adaptation proprement dite qui produit des textes inspirés de l'original, mais parfaitement autonomes. Nous serons ainsi amenés, dans une seconde partie, à examiner cursivement deux éditions françaises assumant l'héritage, au moins partiel, de la Fiammetta: les Angoysses douloureuses qui procedent d'amours, d'Hélisenne de Crenne, et la traduction faite par Maurice Scève du roman espagnol Breve tractado de Grimalte y Gradissa.

On ne pourra perdre de vue l'importance des choix éditoriaux, toujours soucieux de répondre à la curiosité d'un public dont le goût s'oriente résolument, dans le deuxième quart du XVI siècle, vers les fictions sentimentales.

Si surprenant que cela puisse paraître, l'histoire des traductions françaises de l'Elegia di madonna Fiammetta se limite à la période allant de 1531 à 1622. Dans cette histoire, on peut distinguer deux moments: le pre- 
mier, couvrant la décennie 1531-1541 qui voit paraître 5 éditions; le second, marqué par la publication, en 1585, de la traduction de Gabriel Chappuys, et se prolongeant par 3 rééditions de cette même version $(1609,1619,1622)$.

Pour mieux situer l'apparition de la Fiammetta dans le paysage culturel français, on rappellera que si le texte de Boccace comportait assez peu d'éléments romanesques, il donnait en revanche à une voix féminine l'initiative d'un discours essentiellement élégiaque où l'héroöne exhalait ses plaintes de se voir abandonnée par un amant peu soucieux de revenir auprès d'elle, en dépit de ses promesses. Le discours féminin y prenait des tonalités douloureuses, et conduisait Fiammetta à envisager d'abord un suicide (raté), puis à se résigner à une attente qui, en définitive, laissait son histoire ouverte à une "suite». Les éditions italiennes n'avaient pas manqué dès la fin du XV siècle, et leur régularité ne se démentira pas tout au long du siècle suivant.

Nous ne rappellerons pas ce qui est bien connu, à savoir que parmi les œuvres de Boccace pénétrant en France figurent des traductions faites non à partir de l'original en italien, mais de sa traduction en latin. Au premier plan, par le nombre des éditions, figure la version française de la nouvelle d'amour tragique de Guiscardo et Ghismonda (Decameron, IV, 1), donnée en vers par différents traducteurs, de 1493 à la deuxième moitié du $\mathrm{XVI}^{\mathrm{e}}$ siècle. L'histoire en était "piteuse", c'est-à-dire digne d'éveiller la pitié, et la participation émotionnelle d'un public était acquise à une fiction amoureuse marquée par la tristesse, les "angoysses", la cruauté de l'amour dont les joies sont soumises à la brièveté et aux retournements de fortune. Un autre texte connut un notable succès, lié à une perspective plus féminine de l'amour tragique: la nouvelle d'Enea Silvio Piccolomini, Historia de duobus amantibus, traduite en vers par Octavien de Saint Gelais, en 1493, et réunie dans une même édition à la traduction en vers de la nouvelle de Boccace ${ }^{1}$. L'amour féminin se voyait ici opposé à l'égoïsme masculin, et s'adressait au public des "dames», dans un contexte d'émancipation féminine touchant essentiellement les couches aristocratiques ou privilégiées de la société française. D'autres traductions, cette fois à partir des originaux en langue italienne, attestent l'intérêt croissant pour des textes dont la matière essentiellement amoureuse rencontre, et développe sans aucun doute le goût pour les subtilités sentimentales².

1. Gustave Reynier, Le Roman Sentimental avant l'Astrée, Paris, Armand Colin, 1908, p. 20$21 ; 28-29$.

2. Lionello Sozzi, Boccaccio in Francia nel Cinquecento, Genève, Slatkine Reprints, 1999 [1971], p. 54-56, où est signalée l'édition, en 1531, à Paris, des Treize elegantes demandes d'amours, 
On conçoit que la publication de la Fiammetta en français en 1531 ait pu apparaître à l'éditeur particulièrement favorable, et susceptible de trouver son public. Cette première traduction, anonyme, inaugure une décennie où cette version sera reprise, à quelques détails et corrections près, par les quatre éditions suivantes. L'histoire de ces éditions a été retracée dans un article de W.Kemp, qui fournit en particulier des informations inédites sur deux d'entre elles ${ }^{3}$.

Nous pouvons résumer ainsi l'histoire de ces éditions. La première date de 1531, et a été publiée à Paris pour Jean Longis: le titre et l'"Appel aux nobles dames» de Fiammetta qui prélude à son récit manquent dans l'unique exemplaire conservé (New York Public Library). Seuls les six premiers chapitres du texte original ont été traduits. L’année suivante (1532), le même Jean Longis la fait réimprimer sous le titre "Complainte tres piteuse de Flammette a son amy Pamphile. Translatee Ditalien en vulgaire Françoys", reprenant probablement, mais sans certitude, le titre absent de 1531. Cette édition comporte des erreurs et des coquilles par rapport à la précédente.

Cette même année 1532, deux éditions paraissent à Lyon. La première, pour le compte de François Juste, suit fidèlement la version de 1531 (Longis), et reprend le titre de la réimpression de 1532 (Longis). La seconde est celle de Claude Nourry, et son titre differe des précédents: "Complainte des tristes amours de Flammette a son amy Pamphile». Il est précédé d'un titre principal, en gros caractères et en gras: «Flammette», et fait apparaître en deuxième ligne et en gras: «Complainte des tristes amours». Cette édition se signale par le soin apporté au travail éditorial, au niveau de la ponctuation et de la restitution de majuscules, qui la rend plus lisible que les versions précédentes. W. Kemp émet l'hypothèse que cette amélioration du texte serait due à Maurice Scève, lequel aurait remis à Nourry un exemplaire annoté de la première édition parisienne $e^{4}$.

Enfin, en 1541, une nouvelle édition parisienne, chez Denys Janot, reprend la version de 1531 (Longis), avec le titre de 1532 (Longis), en le

extraites du Filocolo de Boccace, choix de dilemmes amoureux caractérisés par l'«analisi sottile dei sentimenti e del cuore" (p. 55). Signalons aussi l'édition, en 1534, à Paris, de l'Hecatomphile, traduction de l'Ecatonfilea de Leon Battista Alberti, où la casuistique amoureuse est également présente; voir Pierre Jodogne, "La diffusion française des écrits de Leon Battista Alberti", in Mélanges à la mémoire de Franco Simone: France et Italie dans la culture européenne, I: Moyen Age et Renaissance, Genève, Slatkine, 1980, p. 181-197.

3. William KeMP, "Les éditions parisiennes et lyonnaises de la Complainte de Flammette de Boccace (1531-1541)", in Studi Francesi, XXXIII (1989), p. 247-265. L'article donne un descriptif détaillé des éditions, sans aborder la question de la qualité de ces traductions.

4. Art. cit., p. 262-263. 
complétant par «le tout reveu et corrigé», bien que les corrections soient peu conséquentes. Avec elle, s'achève cette décennie de traductions de la Fiammetta 5 .

L'examen de ces traductions appellent divers commentaires qui doivent avant tout tenir compte de la confrontation avec les éditions italiennes qui ont pu servir de texte de référence. H. Hauvette est le seul à avoir proposé une telle confrontation, du reste cursive, entre une édition italienne sans date (fin $X^{e}$-début $X V I^{e}$ ?) et l'édition Nourry, pour mettre en évidence «l'insuffisance» de la traduction française, médiocrité à laquelle serait imputable l'oubli où tomberait la Flammette après $1532^{6}$.

Avouons que nous sommes bien empêchés de répondre aisément à toutes les questions posées par ces traductions. D'abord, celles relatives au traducteur, anonyme, et à la qualité de sa connaissance de l'italien?. Hauvette, relevant les nombreux contresens et bévues accumulés, considère que celle-ci devait être imparfaite, tout comme celle qu'il avait des réalités toponymiques et de la mythologie, mais concède qu'il travaillait sur une édition italienne de mauvaise qualité (l'édition s.d. citée). Ce point est évidemment important. Les éditions italiennes de la Fiammetta sont nombreuses dès l'apparition de l'imprimerie. Parmi celles-ci, on relève l'édition princeps de 1472 à Padoue, avec un titre en latin ( $A d$ Flamettam Pamphyli amatricem (ibellus) ${ }^{8}$, et sa réimpression à Naples en

5. L. Sozzi, Boccaccio in Francia..., cit., p. 56, signale une édition lyonnaise d'Arnoullet, sans en préciser la date. W. Kemp (Les éditions parisiennes..., cit., p. 263-264) émet des doutes sur l'existence de cette édition ( «Il est possible que cette édition n'ait jamais existé et qu'il s'agisse d'un enregistrement fautif de l'édition Nourry»), mais ne tranche pas, proposant même l'année 1535 ou "une date ultérieure" pour cette hypothétique parution.

6. Henri HauvetTe, "Les plus anciennes traductions françaises de Boccace", in Bulletin italien, tome $8, n^{\circ} 1$, janvier-mars 1908 , p. 1-17, p. 6: «Il est permis de penser que l'insuffisance de la traduction valut à cette œuvre ardente et passionnée un accueil plus réservé que celui sur lequel les éditeurs avaient compté». L'édition sans date à laquelle fait référence Hauvette est celle conservée à la Bibliothèque Nationale (Rés. Y2306).

7. La traduction dans son ensemble est médiocre. Le traducteur n'avait pu lire, à la date de 1531, les conseils qu'Étienne Dolet dispensait aux traducteurs dans La manière de bien traduire d'une langue en une autre, publié par lui-même en 1540, à Lyon. Il y préconisait la liberté vis-à-vis du mot à mot, la priorité donnée au rendu de l'idée et à la qualité du style et de la syntaxe. Mais la connaissance des principes n'a pas forcément toujours le résultat pratique escompté dans un domaine aussi délicat que la traduction.

8. IOHANNIS. BOCHACII. VIRI. EL-//LOQUENTISSIMI. ADFLAMET-/TAM. PANPHYLI. AMATRICEM// LIBELLUS. MATERNO. SERMO//NE. AEDITUS: INCIPIT: PROLO//GUS. ARTIFICIOSUS.//M. CCCC. LXXII.//DIE. XXI. MAR//CII. FUIT. FI//AME//TE.//BAR. VAL. Patauus.F.F.// Martinus de septe arboribus Prutenus (Cf. Claude BARMANn, Bibliographie des livres localisés imprimés en France au XVIème siècle, tome 4, Paris, 1996). 
1480. Le titre change (Il libro di Madonna Fiammetta) dans les éditions suivantes parues à Venise (1481; réimpression $\left.1491^{9}\right)$. L'édition de Florence (1517, Philippo di Giunta) simplifie le titre: Fiammetta ${ }^{10}$.

Nous avons examiné tout particulièrement deux éditions italiennes: la première est celle que mentionne Hauvette, sans date, et la seconde, celle de Venise (1491). Ce qui rapproche la première de la seconde, outre le titre (s.d.: Incomincia il libro di madonna Fiammetta da lei mandato alle innamorate donne), ce sont un certain nombre de fautes communes à l'une et à l'autre:

accordatore delle cithare di Parnoso [...] ora per Danae ${ }^{11}$

Donna come tu sai poco di la dal piacevole monte falerno in mezzo dellantiche Cumine e di piozuolo: sono le dilettevoli Baye [...] Quivi vicine le isole pietaguse e di conigli abundante e la sepoltura del gran Mileno dante via arengni di plutone [...] e le pestine el monte barbaro varie fatiche dello iniquo Nerone ${ }^{12}$.

Toutefois, l'édition sans date corrige des fautes de 1491: «di Didone» au lieu de "diodone ${ }^{13}$ ». On peut avancer l'hypothèse que cette édition sans date est postérieure à celle de 1481, peut-être à celle de 1491 qui est une réimpression de la précédente, et a dû paraître vers la toute fin du $X^{e}$ siècle, ou au début du siècle suivant.

Le traducteur français a pu utiliser l'édition sans date, moins fautive en certains endroits que celle de 1491, mais il faut admettre qu'il se trouvait parfois en présence d'un texte italien corrompu difficile à interpréter, comme en témoigne le passage correspondant à la toponymie malmenée des environs de Naples, citée précédemment:

[...] et pres de Picheol sont les delectables et naturelz baingz sur la rive de la mer [...] Pres de la est une isle abondante de conilz, en laquelle est la noble sepulture du grand

9. Il libro di Madonna Fiammetta, Maximo de Papia, Venesia, M. CCCC. L XXXXI. Titre complet, en majuscules, en première page: «Commentia illibro ditato a fiammetta da essa alla inamorata mandato explecto per lo illustre poeta et oratore gravissimo giovanni boccaccio ». En dernière page : «finisce il libro di madonna Fiammetta alamorose donne mandato [...]». L'exemplaire est à la Bibliothèque Nationale (Rés - Y2 - 968).

10. Sur ces éditions et les problèmes soulevés par l'établissement du texte, voir Elegia di Madonna Fiammetta, ed. C. Delcorno, in Tutte le opere di Giovanni Boccaccio, ed. V. Branca, vol. V, 2, Milan, Mondadori, 1994, nota al testo: p. 193-215. Le texte de Delcorno est notre texte de référence (dorénavant: Elegia).

11. Elegia, I, 17, p. 41 : «accordatore delle cetere di Parnaso [...] ora per Danne».

12. Elegia, V, 16, p. 98 : «Donna, come tu sai, poco di là dal piacevole monte Falerno, in mezzo dell'antiche Cumme e di Pozzuolo, sono le dilettevoli Baie [...] Quivi vicine le isole Pittaguse e Nisida di conigli abondante, e la sepoltura del gran Meseno, dante via a'regni di Plutone [...] e le Piscine, e Monte barbaro, vane fatiche dello iniquo Nerone».

13. Elegia, I, 17, p. 42 : «di Didone». D’autres remarques en ce sens dans HAUVETTE, «Les plus anciennes traductions françaises...", cit., p. 4. 
Milon qui fust roy Ditalye, lequel donna voyes au royaume de Pluto [...] et palestres, et le mont Barbar, ou est tormente de diverses peines le cruel Neron ${ }^{14}$.

Dans l'édition de 1541 (Janot), qui se présente comme revue et corrigée, mais reprend, rappelons-le, la version de 1531, on trouve, pour ce passage, quelques ajouts parfaitement inutiles («Milon qui fust roy $d u$ pays Ditalye, lequel donna voyes et chemins au royaume de Pluto [...] de diverses peines et travaux» $)^{15}$. Cette observation confirme l'un des aspects les plus remarquables des éditions françaises de la décennie 1531-1541: il s'agit d'une unique traduction, celle de 1531, reprise, en dehors des corrections apportées à la ponctuation par l'édition Nourry (1532), déjà signalées, et des quelques variantes de l'édition Janot (1541), par tous les éditeurs, parisiens et lyonnais. Le travail effectué par Maurice Scève, selon l'hypothèse de W. Kemp, sur l'édition de 1531, visant à améliorer la lisibilité du texte, n'a donc comporté aucune intervention sur la traduction elle-même, laissant en l'état un texte fautif en maints endroits, ou caractérisé par un certain nombre d'omissions.

Avant de signaler les plus notables omissions de cette traduction, précisons que le nom de Boccace n'apparaît dans aucune des éditions de la décennie 1531-1541 : ni dans la page de titre, ni à la dernière page. Or, les éditions italiennes que nous avons citées comportent le nom de l'auteur, hormis l'impression sans date qui n'en fait aucune mention. Il se peut que le traducteur ait travaillé sur celle-ci, car Boccace n'était pas un inconnu pour le public français, depuis la première édition des Cent Nouvelles en 1485. Il est probable qu'on ait tout simplement ignoré quel en était l'auteur, et que cela n'ait eu aucune importance quant à conférer au texte un intérêt supplémentaire.

L'effet d' "accrochage» est confié au titre. Nous avons vu qu'il oscillait entre Complainte tres piteuse de Flammette a son amy Pamphile (Longis, 1531, 1532; Juste, 1532; Janot, 1541) et Complainte des tristes amours de Flammette a son amy Pamphile (Nourry, 1532). W. Kemp suggère que «complainte» traduit «elegia» figurant dans le prologue de la plupart des éditions italiennes ${ }^{16}$. Celles que j'ai citées précédemment ne donnent pas "elegia», mais généralement «il libro». Le mot "complainte» récupère le registre élégiaque perceptible dès le prologue adressé aux dames:

14. Complainte des tristes amours de Flammette a son amy Pamphile, Lyon, Claude Nourry, 1532, fol. lv.

15. Complaincte trespiteuse de Flamette à son amy Pamphile, translatée d'Italien en vulgaire Françoys, le tout reveu et corrigé, Paris, Denis Janot, 1541, fol. lxxiiii.

16. Les éditions parisiennes..., cit., p. 253. 
Les peines et douleurs des misérables croissent abondamment quand ilz congnoissent ou sentent que aulcun en a compassion, et pource que icy plus grand desir de me douloir que daultre chose, et affin que par longue usance et coutume de ce faire ne amoindrisse ma douleur, mais quelle se avance et croisse, il me semble, nobles dames, qui avez les cueurs en amours plus heureuses que moy, quen vous racomptant mon misérable cas et infortune, prendrez de mon malheur quelque compassion ${ }^{17}$.

Le mot "complainte» était fréquent dans les titres de nombreuses pièces poétiques de la même période (la Complaincte que fait l'Amant à sa dame par amours, Complaincte du Prisonnier d'amours) ${ }^{18}$, et se justifie amplement pour un texte dans lequel l'héroïne exhale la longue plainte d'un amour déçu. La "compassion " recherchée par Fiammetta auprès des «nobles dames» apparaît également dans l'un des deux titres ("Complainte tres piteuse»), remplacé dans le second par "Complainte des tristes amours", plus explicite, non seulement quant au contenu élégiaque, mais encore quant au caractère douloureux de l'amour. Ajoutons enfin que le choix d'un titre où l'adresse aux dames amoureuses ("alle innamorate donne») est remplacée par celui de l'amant ("a son amy Pamphile») a le mérite d'y faire figurer un couple amoureux, plus courant dans les titres de la même période, et rappelant les couples célèbres de la littérature sentimentale. Les choix éditoriaux sont intervenus dans le sens d'un titre susceptible de séduire un public, plus féminin que masculin - la «complainte» émane d'une femme - dont les goûts et la sensibilité allaient aux histoires d'amours "pitoyables» auxquelles il pouvait compatir.

La traduction française se caractérise par deux autres aspects notables: les interpolations, et les coupures. Ce sont là deux défauts, nullement étrangers à d'autres traductions de la même période, qui dérogent au principe de fidélité et de respect du texte auxquels nous sommes aujourd'hui (en principe) attachés. Prenons deux courts exemples pour illustrer le premier. Au chapitre I, Fiammetta évoque les changements que le coup de foudre des premiers regards échangés avec Panfilo a provoqués en elle, puis les sentiments qu'elle éprouve à revoir le jeune homme en public. Le traducteur intercale entre ces deux moments ce passage de son cru:

Je croy veritablement que jamais lamour de Tisbée envers Pyradmus ne fut plus fervente, combien que par la grad ardeur damour sexposast au péril et dangier de la mort. Car pour lamour de celluy en qui avoys mis mo cueur nestoit chose au monde que je ne feisse liberallement jusques a vouloir mourir pour luy si loccasion de la mort

17. Complaincte trespiteuse..., cit., [éd. Janot, 1541], fol. ii. Le passage traduit la première phrase du Prologue (Elegia, p. 23).

18. Le roman sentimental..., cit., p. 11. 
se fust offerte a moy tant estoys de son amour esprinse comme je montroys par indices evidentz, gestes exterieurs, et manieres de dire, et de faire declaratives de ma tres vehemente amour ${ }^{19}$.

Cette intercalation introduit la comparaison avec les amours mythologiques de Pyrame et Thisbé dont il n'est question que dans le chapitre VIII de l'Elegia, chapitre d'ailleurs non traduit, comme nous le verrons. Le traducteur développe ici une générosité de sentiment (la jeune femme est prête à sacrifier sa vie pour lui) à partir d'une brève allusion précédente où Fiammetta dit que l'amour a changé son «avarice, naturelle aux femmes» en libéralité toute matérielle. Il est difficile d'apprécier les motifs de cette intervention du traducteur: souci de récupérer les allusions au couple légendaire dont il sacrifie le passage qui le concerne au chapitre VIII, ou simple désinvolture justifiée par le désir d'introduire des codes psychologiques plus familiers au lecteur? Citons une autre intervention du traducteur dans le corps du chapitre V, où Fiammetta décrit une scène au bord de la mer. Il s'agit de jeunes filles qui, en ramassant des coquillages:

montraient souvent les secretz et plaisirs de leurs delicates mammelles plantées en leurs poictrines, les unes par simplicite naturelle, les aultres par ingenieuse et artificielle cautelle, pour prendre les cueurs des jeunes hommes, comme lon prenoit la, par joly passetemps les petits poissons, aulcuns a la ligne, aultres aux retz et filletz, et aultres manieres de pescher ${ }^{20}$.

Cet exemple montre deux choses quant à la personnalité du traducteur: d'une part, une compréhension approximative du texte italien, passablement malmené («uberifero petto» traduit par «mammelles plantées en leurs poictrines»), et d'autre part, la tendance à l'extrapolation, comme le montre la comparaison entre les appâts féminins délibérément offerts aux désirs des jeunes gens et la pêche aux poissons.

Toutefois, l'aspect le plus notable de cette traduction est son caractère incomplet. On relève deux omissions importantes. La première concerne le long passage du chapitre $V$ où Fiammetta décrit les joutes d'armes données à Naples, et où elle compare les participants aux héros grecs d'Homère et de Virgile ${ }^{21}$. Cette description est chargée de références

19. Complainte des tristes amours..., cit., [éd. Nourry] fol xiii. L'interpolation intervient dans le texte italien après " $[\ldots]$ posta in cotale caso " (Elegia, p. 35).

20. Complaincte trespiteuse..., cit., [éd. Janot]. Cf. Elegia, p. 111: «sovente le nascose delizie dello uberifero petto mostravano. E in alcuna altra con più ingegno, altri con reti, e quali con più nuovi artificii, alli nascosi pesci si vedevano pescare».

21. Elegia, p. 112-117 (de "La nostra città, oltre a tutte l'altre italiche [...] " jusqu’à « [...] e lui essere lontano ricordandomi»). 
mythologiques essentiellement empruntées à l'Énéide et aux Métamorphoses. Celles-ci ont-elles découragé le traducteur, ou a-t-il estimé que le passage pouvait être expurgé sans dommage pour la progression psychologique de l'action?

La seconde omission est autrement plus conséquente. En effet, la traduction s'arrête à la fin du chapitre VI, celui où, après sa tentative de suicide manquée, Fiammetta adresse à Panfilo quelques lettres qui resteront sans réponse, puis décide de se rendre, sous couvert de pèlerinage (habile prétexte fourni au mari peu méfiant), dans la ville toscane où le père de Panfilo a rappelé son fils. Ainsi, le chapitre VII, où Fiammetta est à nouveau victime d'une fausse joie et d'une nouvelle déconvenue, le chapitre VIII, où elle compare ses amours à celles des héroïnes de la mythologie et de l'histoire, et le chapitre IX, qui est l'envoi du livre aux dames, sont-ils absents de cette traduction. L'ensemble des passages et chapitres non traduits représente environ un cinquième du texte original.

Hauvette suggérait l'explication suivante: le traducteur, conscient de son insuffisance dans la matière mythologique, n'aurait pas voulu courir le risque de s'embrouiller avec le catalogue d'érudition du chapitre VIII, et aurait considéré qu'avec la finale du chapitre VI l'action avait atteint son acmé $^{22}$. On peut, en effet, admettre que, même si le public n'était pas rebuté par les allusions mythologiques, disséminées çà et là dans le texte pour lui donner une coloration héroïque, l'érudition développée sous la forme d'un inventaire au chapitre VIII, sans intérêt direct pour la progression psychologique du discours ou du récit, ait pu paraître fastidieux pour le lecteur. Le traducteur, et probablement le responsable de la première édition, Longis, ont délibérément proposé un texte épuré, recentré sur les aspects les plus directement émotionnels.

On peut aussi admettre que l'intensité du chapitre VI où le drame passionnel de Fiammetta connaît son acmé, avec le désir de mort, le suicide manqué, le projet de départ pour retrouver Panfilo, justifie la suppression du chapitre VII, qui peut paraître redondant, car il comporte avec une nouvelle fausse joie, une nouvelle désillusion, sans entamer l'espoir de Fiammetta de rejoindre Panfilo. Cette version tronquée, peu respectueuse du texte, pouvait donc se suffire à elle-même, comme le prouvent les trois éditions de 1532 qui tablaient sur un succès d'autant plus assuré qu'elles

22. "Les plus anciennes traductions françaises...", cit., p. 3: l'explication est à rechercher dans "l'affaiblissement de l'intérêt, qui ne pouvait guère se soutenir au diapason où l'ardente passion de l'héroïne l'avait élevé dès le début, et surtout dans le caractère purement historique et mythologique du chapitre VIII ». 
proposaient une fiction sentimentale, pathétique, bien dans le goût du public. Toutefois, ce succès ne fut-il pas assez réservé pour qu'il faille attendre ensuite presque dix ans avant une nouvelle réimpression (Janot, 1541)? Faut-il invoquer ici la désaffection pour une histoire dépourvue d'éléments romanesques, aventureux ou chevaleresques, appréciés par un public accueillant avec empressement les histoires d'amour parsemées d'incidents et d'obstacles ou comportant un dénouement tragique? On peut alléguer, au regard des œuvres à succès de la même période, l'absence d'action extérieure, l'inconsistance des personnages secondaires: le mari et l'amant. Le roman d'Hélisenne de Crenne, Les Angoysses douloureuses qui procedent d'amours, paru en 1538 chez Denis Janot, qui réimprime 3 ans plus tard la traduction de la Fiammetta, et la Deplourable fin de Flammette, traduction de l'espagnol par Maurice Scève, parue à Lyon en 1535 chez François Juste, suggèrent que les libraires escomptaient, par ces «adaptations" plus romanesques du texte de Boccace, une relance du succès éditorial que l'histoire de Fiammetta avait amorcé.

Presque un demi siècle plus tard, en 1585, paraît une nouvelle traduction, par Gabriel Chappuys, avec le titre: La Fiammette amoureuse de M. Iean Boccace gentil-homme florentin: contenant, d'une invention gentile, toutes les plainctes et passions d'amour. Faicte Françoise et Italienne, pour l'utilité de ceux qui desirent apprendre les deux langues, par G.C.D.T. [Gabriel Chappuys de Tours], Paris, chez Abel L'Angelier. Cette traduction sera réimprimée trois fois à Paris, sans changements notables ${ }^{23}$ malgré l'avertissement qui suit le titre ( et mise en meilleur ordre en ceste nouvelle édition»), en 1609 chez Matthieu Guillemot, en 1619 (L'Angelier), et en 1622 ("en la boutique de l'Angelier, chez C. Cramoisy»), dans le même format in $12^{\circ}$ de l'édition de 1585 .

Quelques remarques générales s'imposent. Tout d'abord, nous ne sommes plus en présence d'un traducteur anonyme, mais d'un traducteur extrêmement actif, dans la deuxième moitié du XVI siècle (de 1574 à 1613), qui a travaillé pour des éditeurs lyonnais, puis pour des éditeurs parisiens. Chappuys a largement contribué à la diffusion de la littérature italienne en France (Doni, l'Arioste, Castiglione...), tout en élargissant son activité aux traductions d'œuvres espagnoles ${ }^{24}$. Ces traductions ont

23. Les innovations apportées à l'édition de 1585 par les suivantes consistent dans la traduction des rubriques en marge du texte italien, et dans l'adjonction d'un «avertissement au lecteur».

24. Sur la personnalité et l'activité de Gabriel Chappuys, je recommande l'excellent numéro 6 de la revue Filigrana: La Lettre, le Secrétaire, le Lettré: de Venise à la cour de Henri III, 2 vol., 
souvent été soumises à des jugements sévères (tendance à la dilution), mais c'est un traducteur qui connaît bien la langue italienne, et avec lui on peut affirmer que la traduction de la Fiammetta fait peau neuve.

En effet, sa traduction, présentée en regard du texte italien, est juxtalinéaire. Les paragraphes sont isolés par des blancs qui rendent très lisible le passage d'une langue à l'autre. Elle répond à son objet qui est de faciliter au lecteur français l'apprentissage de la langue italienne, même si l'on peut émettre des réserves quant à le faciliter avec un texte à l'écriture aussi complexe que celle de la Fiammetta. L'intention d'être utile à "ceux qui désirent apprendre les deux langues » est étonnante, car elle laisse entendre soit que les lecteurs français ont besoin de parfaire la connaissance de leur langue natale, soit que la traduction s'adresse aussi aux Italiens ${ }^{25}$. L'intention pédagogique clairement affichée est peut-être l'une des raisons justifiant les trois rééditions successives, dans un milieu où l'italianisme est très largement présent dans la France de Henri IV, et dans celle de la régence de Marie de Médicis.

Cette traduction a un autre mérite par rapport aux précédents : elle est intégrale. Le principe adopté d'une traduction juxtalinéaire n'explique pas tout. Sur les 26 éditions italiennes (il s'agit d'une approximation) que j'ai pu répertorier pour le $\mathrm{XVI}^{\mathrm{e}}$ siècle, celles qui ont été éditées quelques années avant la traduction de Chappuys présentent une qualité certaine. La traduction de Chappuys est en conformité avec le texte établi dans ces éditions récentes, comme le montre le texte en regard ${ }^{26}$. La table présente 7 livres (ou chapitres), mais en regroupant les chapitres III et IV sous un seul livre, et les deux derniers sous le livre VII, table conforme aux éditions italiennes depuis 1527 qui ne distinguaient pas l'épilogue du chapitre

Grenoble, Ellug, 2000-2001. En particulier, Patrizia De CAPITANI, «Un traducteur français de textes italiens à la fin de la Renaissance. Gabriel Chappuys (env. 1546 - env. 1613)», vol. I, p. 89114, et Patrick MulA, "De Venise à Paris. L'art des secretaires de Gabriel Chappuys: entre traduction et création", vol. I, p. 115-182.

25. Le principe de l'édition bilingue n'était pas nouveau: Gabriel Chappuys avait donné en 1583, avec texte italien en regard, les Dialogues philosophiques... de Giambattista Giraldi Cinzio, chez l'Angelier, et, en 1585, le lecteur curieux de l'apprentissage de l'italien peut consulter sa traduction de Castiglione: Le parfait courtisan du comte Baltasar Castillonois es deux langues respondans par deux colomnes..., Paris, N. Bonfons, 1585.

26. L'édition ayant servi de base à la traduction de Chappuys pourrait être la suivante: L'amorosa Fiammetta di M. Giovanni Boccaccio di nuovo corretta et ristampata con le postille in margine et con la tavola nel fine delle cose notabili. In Vinegia, appresso Iacomo Vidali, M. D. LXXV. Voir aussi Dario RASTELLI, "Notizie storiche e bibliografiche sulla composizione e sulla fortuna dell'Elegia di Madonna Fiammetta e del Ninfale Fiesolano di G. Boccaccio ", Annali della Biblioteca Governativa e Libreria Civica di Cremona, 1951, 2, p. 3-22. 
$\mathrm{VIII}^{27}$. Le titre lui-même (La Fiammette amoureuse) est partiellement conforme au titre italien (L'amorosa Fiammetta), mais développe ce dernier: contenant, d'une invention gentile, toutes les plainctes et passions d'amour, une manière de récupérer le contenu élégiaque et fortement sentimental du récit. Enfin, voilà une œuvre qui retrouve son auteur aux yeux du public français (La Fiammette amoureuse de M. Iean Boccace gentilhomme florentin). Le lien est désormais fait entre l'auteur de la Fiammetta et les prestigieuses traductions qui avaient établi la célébrité de l'écrivain italien, en particulier celle du Decameron par Antoine Le Maçon en $1545^{28}$.

La qualité globale de la traduction est bonne. Le principe de l'édition bilingue maintient la liberté du traducteur dans de raisonnables limites, même si la tendance à rendre souvent un terme par deux équivalents peut paraître contestable pour une traduction qui se veut pédagogique ${ }^{29}$. Le traducteur anonyme usait du même procédé qui relève d'une habitude stylistique courante dans la littérature de l'époque. Si l'on veut démontrer la supériorité incontestable de la traduction de Chappuys sur celle de son prédécesseur, il suffira de confronter les passages que nous avons cités plus haut. Le passage si malmené de la description du paysage napolitain devient chez Chappuys, aidé par un texte italien correct et non corrompu:

un peu de là le plaisant mont Falerne, au milieu de l'antique Cume, \& de Pozzuol, est la plaisante ville, Baye, sur le rivage de la mer [...] Pres de là est aussi l'isle de Pitacuse, \& Nitide, abondante en connils, \& lievres, \& la sepulture du grand Misene, qui mène aux royaumes de Pluton [...] \& le theatre (lieu commun des anciens ieux) $\&$ les Piscines, \& le mont barbare, vains travaux $\&$ ouvres de l'inique $\mathrm{Neron}^{30}$.

Comparons également ces deux extraits. Le premier appartient à l'édition Nourry et le second à la version de Chappuys:

27. Ainsi la Table des choses contenues en l'œuvre de l'édition de 1585 [et suivantes] traduit-elle la "tavola nel fine delle cose notabili» de l'édition italienne de 1575 (L'amorosa Fiammetta..., op. cit.) : «Songe de la Fiammette, auquel toute son infortune avenir est demonstree» («Sogno della Fiammetta nel quale tutta la sua futura infelicità si dimostra»).

28. La première édition fut imprimée, à Paris, par Estienne Roffet, et rééditée trois ans plus tard par le même. Dans sa dédicace à Marguerite de Navarre, le traducteur écrit: «Et mes raisons estoient que Bocace avoit esté, comme j'ay tousiours ouy dire aux plus sçavans, l'homme de toute l'Italie qui a paradventure le mieulx escript en sa langue que nul autre fit oncques, voyre jusques à soustenir que Ciceron ne Demosthene n'avoient point mieulx ne plus proprement et aysement parlé, l'un en latin et l'autre en grec, que Bocace avoit fait en tuscan"; in Jean BocCACE, Le Décaméron, traduction de Le Maçon, Paris, Flammarion, Librairie des Bibliophiles, s.d., tome 1, p. 4.

29. Par exemple, «non vi conforto» devient «je ne vous conseille et advise», et «in non commettervi» est rendu par «à ne vous commettre et fier».

30. La Fiammette amoureuse..., cit., p. 201-202; Elegia, p. 98 (texte déjà cité). 
Plus je pensoys que iestois plus contente, si contente on peut estre, de mal avoir, par ce que avoys ayme fidellement et que leaulte est tout le bien quon peult trouver en amours $^{31}$.

Et puis en blasmant une telle pensee, ie disois: Ie suis plus contente (si l'on peut toutesfois estre contente de mal avoir) d'avoir fidelement aymé ${ }^{32}$.

Le traducteur anonyme semble avoir eu du mal avec le texte italien, peu souple à cet endroit, mais a cédé à son habitude d'interpoler une réflexion de son cru (en italiques dans la citation). Chappuys parvient, avec une grande aisance syntaxique, à rendre à l'original une lisibilité dont il semble plutôt dépourvu au premier abord.

Chappuys a fait précéder sa traduction d'un avertissement «Aux nobles et vertueuses dames». Sans texte italien en regard, cet avertissement pourrait passer pour un texte propre au traducteur. Or, il n'en est rien, car l'édition italienne de 1575 donne un avertissement "Alle nobili et virtuose donne» que Chappuys se donne simplement la peine de traduire, contrairement à ce que croyait Hauvette ${ }^{33}$. La teneur de cet avertissement est de nature moralisante. En 1517, le florentin Bernardo di Giunta avertissait en tête de son édition de la Fiammetta que le contenu de cette histoire pouvait servir à éviter de tomber dans les pièges de l'amour. Faut-il pour autant prendre au sérieux ce genre d'avertissement, sinon comme habile procédé "publicitaire» destiné à appâter le lecteur ou la lectrice? Il existe toutefois une différence de ton et d'intention dans l'avertissement traduit par Chappuys en exergue à sa Fiammette amoureuse, et dont je cite un extrait:

L'œuvre de la Fiamette [...] n'a esté imprimée [qu'] afin que de là vous apreniez à fuir ses lascivetez [de l'amour] [...], vous viendrez à cognoistre $\&$ compredre, que les fruicts du vain Amour ne font autre chose que tromperies, tourmes, deshonneur, perte, dommage et mort; mais mort, non tat ignominieuse au monde, que dommageable et pernicieuse à l'Ame. Pour cette cause, l'advertissemet et instruction que vous autres nobles et vertueuses Dames, devez predre de ce petit œuvre, doit estre cete cy, que fuyant les vanitez amoureuses, vous vous apliquiez entièremet à l'amour de Dieu $[\ldots]^{34}$.

31. Complainte des tristes amours..., cit., fol. lviii.

32. La Fiammette amoureuse..., cit., p. 213; Elegia, p. 102 : «Poi dannando cotale pensiero, più essendo contenta, se essere si puote contenta di male avere, sono d'avere fedelmente amato».

33. "Les plus anciennes traductions françaises...", cit., p. 10.

34. La Fiammette amoureuse..., cit., f. ij. Cf. L'amorosa Fiammetta..., cit.: «L'opera della Fiammetta [...] è posta [...] accio che voi di qui impariate a fuggir le lascivie sue [di Amore] [...], verrete a comprendere, che i frutti del vano Amore, altro non sono, che inganni, tormenti, vergogna, danno e morte; ma morte non tanto ignominiosa al Mondo, quanto dannosa all'Anima. Per la qual cosa l'avertimento, che voi Nobili donne, havete a pigliar da questa operina, ha da esser questo, che fuggendo le vanità amorose, v'applichiate interamente all'amor santo di Dio benedetto". 
En effet, il ne s'agit pas seulement de présenter l'histoire de Fiammetta comme une mise en garde contre les périls de la passion amoureuse, qu'il faut sous-entendre d'autant plus grands chez une femme mariée, mais d'en faire un bréviaire pour conduire à l'amour de Dieu, à travers l'exemple à ne pas suivre. Dans un contexte post-tridentin, l'avertissement (qui ne comporte aucune pédagogie de la fidélité conjugale, notons-le!) peut effectivement s'expliquer, et l'on peut penser qu'il fonctionne comme un paravent destiné à faire passer un discours où l'héroïne, qui se voue aux sombres Furies avant de tenter son suicide, illustre le désespoir dans lequel tombent les âmes privées du recours à Dieu.

Toujours est-il que, dans la réimpression de 1609, l'avertissement dont il vient d'être question est suivi d'un "avertissement au lecteur", dont on ne sait s'il émane de Chappuys ou de l'éditeur. Son but est de justifier les corrections et améliorations apportées à la traduction, et on peut y lire:

Tout ce qui vient d'Amour est toujours agréable: sa flamme ne peut produire rien de rude, incorrecte ou mal poly. C'est pourquoi ce Livre a esté si bien reçu en la première impression ${ }^{35}$.

C'est donc bien le langage de l'Amour qui doit parler au lecteur que l'on a précédemment mis en garde contre les effets dévastateurs de cette flamme. Chappuys, si c'est lui, dit ici la beauté d'un texte avec lequel s'établit une relation nouvelle, celui de l'appréciation de la qualité intrinsèque du style. Le langage de l'amour n'est pas seulement le moyen d'apprentissage d'une langue aux séduisants accents. Les trois réimpressions de la Fiammette de Chappuys, en ce début du XVII siècle, semblent s'accorder à des préoccupations éditoriales. Ce qui plait alors dans l'amour romanesque, c'est son caractère grave et douloureux, comme l'explique G. Reynier qui cite de nombreux exemples où la séduction du texte passe d'abord par ses effets tragiques $^{36}$.

Ici prend fin l'histoire des traductions françaises de la Fiammetta, dans la mesure où il n'apparaît pas que ce texte ait été retraduit avant l'essai que nous en avons proposé en $2003^{37}$. L'ombre lumineuse du Decameron a

35. La Fiammette amoureuse..., Paris, Matthieu Guillemot, 1609.

36. Le roman sentimental..., cit., p. 243: "Voilà les effects de l'amour, s'écrie l'auteur des Destinees des Amans (1603), voilà les effects de l'amour qui, soubs je ne sçay quel plaisir, nous donne un tourment qui nous travaille et afflige incessamment: la conscience nous remord et la craincte nous emmene en un estrange desespoir, si bien que l'Amour n'est autre chose qu'un troublement d'esprit, une douleur et alienation de tout entendement». L'avertissement en tête de la traduction de Chappuys ne dit pas autre chose, et dans des termes presque identiques.

37. Fiammetta, traduction nouvelle par Serge Stolf, Paris, Arléa, 2003. 
joué sur l'effacement des autres œuvres de Boccace que l'on continue, assez injustement pour certaines, à qualifier de mineures.

Comme nous l'avions annoncé, nous consacrerons quelques remarques à l'influence que ces traductions de la Fiammetta ont eu sur la production française du XVI ${ }^{\mathrm{e}}$ siècle. Cette question a été traitée par les spécialistes de la littérature française, et nous ne nous y appesantirons pas $^{38}$. Tout d'abord, la traduction par Maurice Scève d'un roman espagnol où l'on voit réapparaître la Flammette est éditée dans la décennie 1531-1541. Ce roman espagnol de Juan de Flores, publié à la fin du XV siècle, a pour titre Breve tractado de Grimalte y Gradissa. Maurice Scève, et c'est un de ses premiers travaux, le traduit en 1535 pour François Juste qui, nous l'avons vu, avait réédité à Lyon en 1532 la traduction de Fiammetta. Le titre français, $L a$ deplourable fin de Flamete elegante invention de Jehan de Flores espaignol traduicte en Langue Françoise ${ }^{39}$, n'a rien à voir avec l'original espagnol, mais il souligne le lien qui unit le roman à Fiammetta. Il est plus que probable que c'est à l'initiative de l'éditeur, escomptant un succès dans la foulée des 3 éditions de Fiammetta en 1532, que Maurice Scève l'a traduit ${ }^{40}$.

Dans son «epistre proemiale» aux lecteurs, Scève dit avoir été poussé à cette traduction pour deux raisons. D'une part, l'attente des lecteurs:

le regret qu'avez de la non finie histoire de Flamete, qui vous tient en désir suspendus, pour vous réconforter

et, d'autre part, la valeur didactique du livre:

vous enseigner a cauteleusement aymer, qui n'a ayme, et saigement desaimer, qui es lacs de ce cruel tyrant d'amour est entreprins ${ }^{41}$.

La première raison, qui seule nous intéresse ici, s'éclaire si l'on sait que le roman espagnol proposait en grande partie la «suite» ou "continuation» de Fiammeta. La femme, Gradisse, a reçu une lettre de Fiammetta

38. Un article intéressant a été consacré à ce sujet par Sergio Cappello, "La non finie histoire de Flammette. Avatars d'un dénouement incertain ", in Il Bianco e il Nero. Rivista di anglistica, germanistica, romanistica e italianistica, 1997, p. 39-53.

39. Édition soignée [Château de Chantilly]. Une réédition paraît chez D. Janot en 1536, à Paris: La deplourable fin de Flamete elegante invention de Jehan de Flores Espaignol traduicte en langue Françoyse, nouvellement imprimee a Paris par Denys Ianot [B.N. RES P - Y2 - 251]. Il en existe également un premier état à la Bibliothèque de Chantilly.

40. Verdun-Louis Saulnier, Maurice Scève (ca. 1500-1560), Genève, Slatkine, 1981 [194849], p. 49-71.

41. La deplourable fin..., [éd. 1535], cit., Epistre proemiale. 
ou a lu son histoire (les deux versions coexistent) sur les conseils de son amant Grimalte. Indignée par la perfidie masculine, elle se déclare incapable de répondre à l'amour que lui voue Grimalte, à moins que celui-ci ne se soumette à une singulière épreuve: retrouver Flammette et Pamphile, et les réconcilier. Voilà Grimalte parti et, au terme de longs voyages, retrouvant la malheureuse Flammette qui a fait le pèlerinage annoncé à la fin du chapitre VI de la Fiammetta. À Florence, ils retrouveront Pamphile qui, malgré de grandes réticences, accepte une ultime entrevue. Celle-ci tourne court pour Flammette, car Pamphile a décidé de mettre un terme à leurs amours. Flammette mourra de douleur sous les yeux de Grimalte. La fin de l'histoire est occupée par le repentir de Pamphile qui se fait ermite pour expier la mort de la malheureuse, et par le désespoir de Grimalte qui va lui tenir compagnie au désert, nu, marchant à quatre pattes!

L'intérêt de cette traduction est que Maurice Scève la propose alors que la version française de Fiammetta circulait dans le public qui, dans la fin anticipée au chapitre VI, pouvait considérer l'histoire comme inachevée, mais riche de potentialités: Fiammetta y annonçait son projet de partir sur les traces de Pamphile. La coupure opérée par le premier traducteur serait donc en partie explicable par l'inintérêt des trois derniers chapitres du point de vue de l'action ${ }^{42}$. Et sans doute l'histoire ne devait-elle pas davantage paraître achevée pour les lecteurs espagnols puisque Juan de Flores en proposait une continuation, à vrai dire plus complexe qu'il ne semble au premier abord. En effet, le roman espagnol exploite une situation romanesque parallèle à celle de Fiammetta et Panfilo: l'histoire de Grimalte et Gradisse, où la dureté de la femme contraint l'amant à un retrait du monde, est l'inverse même de celle de l'héroïne de Boccace. S'agissait-il pour l'éditeur lyonnais, François Juste, de relancer une bonne histoire sentimentale, en exploitant le goût des lecteurs pour les dénouements tragiques et pour cette correspondance tant désirée entre la souffrance de la femme et celle de l'homme, la fin du roman espagnol rétablissant une sorte de loi du talion?

Un mot encore sur le singulier roman féminin d'Helisenne de Crenne, paru chez Denis Janot en 1538, Les Angoysses douloureuses qui procedent

42. Sergio CAPPELlo, "La non finie histoire... », cit., p. 43: «Dans le cas de la traduction anonyme de 1531 on a interrompu le récit au chapitre VI sur l'espoir et l'attente d'un voyage futur. Ainsi ont été éliminés les derniers chapitres où est soulignée avec insistance la non solution du cas et on a construit de par cette interruption un dénouement virtuel positif». 
d'amours ${ }^{43}$. Le rapprochement avec la Fiammetta est particulièrement sensible dans la première partie, comme le montre ponctuellement G. Reynier ${ }^{44}$. Comme Fiammetta, Hélisenne est mariée et s'éprend d'un jeune homme, et sa passion inassouvie pour Guénélic (elle ne pourra se donner à lui) s'épanche en de douloureux accents qui prennent pour confidentes les nobles dames. À vrai dire, la lecture révèle une dépendance toute relative par rapport au texte de Boccace. Hélisenne de Crenne sait habilement démarquer certains éléments de la Fiammetta ${ }^{45}$, sans aliéner jamais son originalité dans la progression du récit et dans le rôle qu'elle donne au mari, source d'un conflit développé jusqu'à la conclusion finale de cette première partie: elle est enfermée par son époux dans la tour d'un château.

Le roman d'Hélisenne donne une grande importance aux lettres échangées entre les deux "amants» (situation absente de la Fiammetta), ce qui donne à penser qu'Hélisenne a fort probablement lu le texte de Enea Silvio Piccolomini, Historia de duobus amantibus, traduit en français dès la fin du XVe siècle, et dont G. Reynier signale une traduction éditée en 1537. L'infléchissement moral qu'on discerne çà et là (dans le titre lui-même où figure une mise en garde "à ne suyvre folle amour») montre la distance prise avec la Fiammetta.

Concernant les traductions de la Fiammetta, nous constatons donc qu'entre les premières éditions italiennes $(1472,1480,1481)$ et la première traduction française, incomplète, de 1531, s'écoule un demi-siècle.

43. Le titre complet est: Les Angoysses douloureuses qui procedent d'amours contenant troys parties composees par dame Helisenne de Crenne laquelle exhorte toutes personnes à ne suyvre folle amour, Paris, D. Janot, 1538.

44. Le roman sentimental..., cit., p. 112-117.

45. Ainsi, Hélisenne reprend-elle en partie la scène de désespoir à laquelle se livre Fiammetta, à la fin du chapitre VI: "Ainsi qu'estois tant travaillée que ne povois plus, voyant qu'impossible m'estoit de parvenir à mon cruel désir de la mort parce que j'avois tousjours seure garde, dont pour ceste cause regardant mes belles mains blanches et déliées, par furieuse rage je commençay à dire: o mains iniques, qui m'avez aornée et m'avez servy selon mon curieux désir pour complaire à celuy que j'ayme si ardemment. Pour lequel, par estre privée de sa veue, suis réduite jusques à l'extrémité; vous avez esté cause en partie de mon mal, à quoy vous imposerez fin en transperçant ce cueur variable qui s'est laissé surprendre et vaincre d'amour" (HéLISENNE DE CRENNE, Les angoysses douloureuses qui procèdent d'amours (Première partie), présentation par J. Vercruysse, Paris, Minard, 1968, p. 146). Cf. Elegia, p. 155: "per che con romore a gridare cominciai: "O mani inique, e possenti ad ogni male, voi, ornatrici della mia bellezza, foste gran cagione di farmi tale che io fossi disiderata da colui, il qual più amo. Dunque, poiché male del vostro uficio m’è seguito, in guiderdone di ciò ora l'empia crudeltà usate nel vostro corpo : laceratelo, apritelo [...]". 
Pendant une décennie (1531-1541), cinq éditions, ce qui n’est ni pléthorique ni indigent. Un autre demi-siècle, ou presque, sans réimpression ni traduction nouvelle, avant celle de Gabriel Chappuys, en 1585, intégrale et bilingue, suivie de 3 réimpressions $(1609,1619,1622)$, puis l'oubli.

Le plus intéressant est probablement l'écho qu'a pu rencontrer, au cours de la décennie en question, l'histoire de Fiammetta, écho dont témoignent de manière divergente la traduction (1535) du roman espagnol de Juan de Flores, suivant trois impressions de la Flammette pour la seule année 1532, et le roman d'Hélisenne de Crenne (1538, mais suivi de trois autres éditions en 1540 et 1541). Les éditeurs apparaissent sensibles à une certaine réception du public français où l'engouement pour la thématique de l'amour douloureux s'accommode, semble-t-il, beaucoup mieux de fictions sentimentales, plus riches en aventures ou en merveilleux. Ainsi, au chapitre XLII de La deplourable fin de Flamecte, Grimalte voit en songe Flamette entourée de monstres hideux, et la suite du roman d'Hélisenne est pleine des exploits chevaleresques de Guénélic. La Fiammetta, dont la grande originalité reposait sur une action entièrement soutenue par l'introspection psychologique, n'a incontestablement pas eu le même succès en France qu'en Italie, à une époque où la langue française n'avait peut-être pas encore coulé ses inflexions dans celles, latines et ondoyantes, de la Fiammetta. On mesurera alors combien une telle traduction peut présenter aujourd'hui de difficultés pour ne pas décourager le public français d'accéder aux beautés de l'Elegia. 